\title{
En busca de la "metodologia mixta" entre un estudio de corte cualitativo y el seguimiento de una cohorte en una encuesta retrospectiva*
}

\author{
Edith Pacheco** \\ Mercedes Blanco***
}

Con el objetivo de presentar un ejercicio que muestre la importancia de la llamada "metodología mixta", el presente artículo constituye un acercamiento exploratorio a la utilización simultánea de fuentes de datos claramente identificadas con los estilos de investigación cualitativo y cuantitativo. Uno de los caminos que encontramos para vincular ambas fuentes fue por medio de la construcción de una tipología -con los datos cuantitativos- similar a la elaborada previamente en el estudio cualitativo, que da cuenta de las posibles articulaciones de cuatro trayectorias vitales (escolar, laboral, conyugal y reproductiva) de un grupo de mujeres de clase media urbana. La búsqueda de diferencias y matices se convirtió en un propósito común para los dos tipos de fuentes de datos utilizados; de hecho, consideramos que el haber conjuntado un recorte de tipo cuantitativo con los resultados previos del estudio cualitativo fue precisamente lo que hizo posible enriquecer a la vez que reforzar la propuesta de la existencia de la diversidad dentro de la homogeneidad. Asi, después de haber confrontado diferentes tipos de fuentes de información, llamó la atención que el conjunto de casos del estudio cualitativo síreflejó los rasgos generales que presentaron universos similares extraidos de la base de datos estadisticamente representativa. Se puede decir que algunas de las características que conformaron el perfil sociodemográfico del conjunto de los casos cualitativos encontraron su correlato en el comportamiento similar de las mismas propiedades de las submuestras cuantitativas. Además, no sólo las caracteristicas aisladas fueron similares, incluso fue posible construir una tipología parecida con las poblaciones ambas fuentes, lo cual refuerza los supuestos de la llamada "metodologia mixta".

Palabras clave: metodología mixta, cohorte, trayectorias vitales, tipología, sectores medios.

Fecha de recepción: 7 de diciembre de 2001.

Fecha de aceptación: 8 de febrero de 2002.

\footnotetext{
* Las autoras agradecen a la actuaria Cristina Mexicano su valiosa colaboración en el manejo de la base de datos estadísticos y en la construcción de los diagramas.

** Profesora-investigadora de El Colegio de México. Correo electrónico: mpache co@colmex.mx.

*** Profesora-investigadora del Centro de Investigaciones y Estudios Superiores en Antropología Social (CIESAS-D.F.). Correo electrónico: merblanco@prodigy.net.mx
} 


\section{Introducción ${ }^{x}$}

Un reto aún vigente en las ciencias sociales en general lo representan la vinculación, articulación y combinación de niveles de análisis y fuentes de información de diversos tipos, con la idea de dar cuenta más ampliamente de cualquier fenómeno bajo estudio. La literatura especializada en el abordaje metodológico dentro de las ciencias sociales rescata así el debate entre los llamados cuantitativistas y los cualitativistas y, a pesar de que reivindica la indispensabilidad de su mutua consideración para lograr análisis más comprehensivos de cualquier fenómeno social, deja claro que aún en la actualidad representa un problema por resolver, sobre todo por lo que se refiere a la práctica de proyectos de investigación sustentados en información empírica de ambos tipos (Hentschel, 1999).

En un texto elaborado previamente (Blanco y Pacheco, 2001) llevamos a cabo una primera aproximación a tan complejo reto partiendo de una de sus tantas facetas. En concreto se trató del manejo conjunto de dos fuentes de información claramente identificadas con cada uno de los polos de la mencionada dicotomía: se eligió una encuesta estadísticamente representativa -la Encuesta Nacional de Empleo Urbano, ENEU-y un conjunto de entrevistas a profundidad realizadas por una de las coautoras (Blanco, en este volumen). La problemática que se abordó entonces, al igual que en esta ocasión, gira en torno al desarrollo de las trayectorias laborales femeninas, tema que, por cierto, inevitablemente nos lleva a otro de los puntos teórico-metodológicos nodales de la investigación social: la consideración de la dimensión diacrónica versus los cortes sincrónicos, que son mucho más frecuentes en la investigación sobre mercados de trabajo en México. Por lo que respecta al manejo teórico y empírico de la di-

${ }^{1} \mathrm{La}$ intención de presentar en un mismo volumen dos artículos que dan cuenta de elaboraciones teóricas y metodológicas relacionadas es proporcionar al lector el seguimiento de un proceso que deriva en la combinación de bases de datos y enfoques analíticos diferentes para, finalmente, cristalizar en la propuesta de una "metodología mixta". Por ello el presente artículo contiene algunos párrafos descriptivos similares, o incluso iguales, a los del texto de M. Blanco que aparece en esta misma publicación. Esto fue necesario porque dichas partes se refieren a la misma base de datos cualitativa que se utilizó tanto en aquella investigación como en el ejercicio metodológico y técnico que aquí se presenta. Además, teniendo en cuenta que algunos lectores podrían estar interesados en leer sólo uno de los artículos, consideramos indispensable hacer referencia en ambos textos a cierta información básica, sobre todo cuando se trata de datos que dan sustento, en cada caso, a las elaboraciones analíticas posteriores. 
mensión temporal, en esta oportunidad hemos recurrido a una de las herramientas clásicas de la demografía, el estudio de cohorte, pero enmarcada en los supuestos teórico-metodológicos del denominado enfoque del curso de vida que justamente asigna a la cohorte de nacimiento una connotación de ubicación espacio-temporal y de tránsito a lo largo de la historia.

En el plano metodológico, el camino trazado por la investigación que se ha desarrollado en México sobre el estudio de las trayectorias laborales $s^{2}$ en parte ha estado condicionado por el tipo de fuentes de información con que cuenta el país, es decir, si bien existe un acervo importante de encuestas de empleo, hasta hace muy poco tiempo no se contaba con historias laborales que permitieran realizar estudios desde una perspectiva longitudinal. En consecuencia, no era posible reconstruir la historia laboral de la población mexicana en su conjunto, de tal suerte que se pudiera estudiar la incorporación al mercado de trabajo de hombres y mujeres partiendo del análisis integral de las trayectorias vitales de los individuos a lo largo de todo el curso de vida (por ejemplo, las trayectorias educativas, reproductivas y conyugales, además de las laborales propiamente dichas). Apenas en 1998 se realizó, por primera vez en México, una Encuesta Demográfica Retrospectiva (Eder) con representatividad nacional, que incorporaremos en el presente ejercicio y de la cual hablaremos más adelante. ${ }^{3}$

Tomando en cuenta todo lo anterior, la propuesta, tanto en ese primer ejercicio que mencionamos con la ENEU como ahora con la Eder, constituye un acercamiento exploratorio a la utilización simultánea de fuentes de datos claramente identificadas con los estilos de investigación cualitativo y cuantitativo, con el propósito de presentar un ejercicio que demuestre la importancia de la llamada metodología mixta. Uno de los caminos que encontramos para vincular ambas fuentes fue la construcción de una tipología -con los datos cuantitativos- similar a la elaborada previamente en el estudio cualitativo, que da cuenta de las posibles articulaciones de cuatro trayectorias vitales (escolar, laboral, conyugal y reproductiva) de un grupo de mujeres de clase media urbana. Esto se hizo con la finalidad de dar segui-

\footnotetext{
${ }^{2}$ En otros textos (Blanco, en este volumen; Blanco y Pacheco, 2001) hemos realizado una revisión bibliográfica, no exhaustiva pero sí representativa, sobre los estudios de trayectorias laborales llevados a cabo en México.

${ }^{3}$ Cabe mencionar que una de las ventajas de la Eder es que se encuentra disponible en internet para la consulta del público en general en la siguiente dirección electrónica: http://www.gda.itesm.mx/cee/eder/proyecto.htm.
} 
miento a algunas de las variables que caracterizan la vinculación familia-trabajo de las mujeres de sectores medios, bajo el supuesto de que dicha combinación de fuentes podría aportar más elementos para el análisis de las trayectorias laborales femeninas y para la implementación práctica de la combinación de fuentes de datos tradicionalmente consideradas opuestas.

Entre las preguntas que han guiado tanto la investigación cualitativa como los ejercicios de combinación de fuentes de datos cabe mencionar las siguientes:

Partiendo del muy conocido hecho de que prácticamente cualquier mujer que lleva a cabo una actividad económica tiene que combinar los ámbitos laboral y familiar/doméstico (la famosa "doble jornada"), ¿cómo se da la interrelación familia-trabajo en mujeres de sectores o clases medias? Más específicamente, ¿cómo se construyen las trayectorias laborales de estas mujeres en combinación con sus trayectorias escolares y familiares? Por último, y representando de alguna manera la pregunta rectora en estos ejercicios, ¿qué tipo de combinaciones se dan en la interrelación familia-trabajo en mujeres de sectores medios?

En el marco de tales preguntas, este trabajo representa un ejercicio metodológico, y no precisamente la presentación de un resultado de investigación. Para lograr este objetivo el texto se ha estructurado en cinco apartados; los dos primeros están dedicados a la discusión teórico-metodológica, el tercero presenta la forma en que se construyó la matriz de datos, base de este esfuerzo analítico y, finalmente, los dos últimos apartados se centran en la tarea de confrontar dos aproximaciones analíticas identificadas con las dos perspectivas que hemos mencionado previamente: la cualitativa y la cuantitativa.

De la oposición "cuali-cuanti" a la propuesta de la "metodología mixta"

Desde principios de la década de 1980 en las ciencias sociales ha estado presente de manera importante el debate -que a veces ha adquirido tintes de una verdadera guerra- entre los enfoques llamados genéricamente "cuantitativos" y "cualitativos" (Salles, 2001). En un texto anterior (Blanco y Pacheco, 2001) hemos recorrido el desarrollo de dicho debate, encontrando su génesis en los supuestos de la sociología del siglo XIX, pasando por el predominio del positivismo durante mu- 
chos años, hasta llegar a las críticas y nuevas proposiciones de los llamados antipositivistas $y$, finalmente, al reconocimiento mutuo y a los intentos de integración. ${ }^{4}$

De entre las propuestas iniciales, provenientes del reconocimiento del "campo enemigo", en los años ochenta destaca la idea de la complementariedad como una vía hacia la reconciliación. De ésta se pasa a la búsqueda de la integración, a la búsqueda de la disolución de la dicotomía "cuali-cuanti", para lo cual autores como Aaron Cicourel (1981) hablan de un "modelo interactivo" que contiene fuentes de datos que se "activan en paralelo". Las propuestas de la segunda mitad de los años noventa pretenden seguir avanzando en esta integración y, entonces, algunos autores hablan de aquello que llaman un "continuum interactivo" (Newman y Benz, 1998).

Teniendo el mismo punto de partida de otros autores antipositivistas -o sea, la asunción de que la realidad es una construcción social-, y manteniendo la afirmación de que los enfoques cualitativos y cuantitativos no sólo no son mutuamente excluyentes sino que existe la necesidad de abandonar tal dicotomía, los autores más recientes centran su propuesta en torno a la pregunta de investigación. Es decir, una vez habiendo establecido claramente que ningún método es "superior" o "mejor" que los otros, por ejemplo, Newman y Benz proponen que el eje de la posibilidad de elección y combinación de enfoques sea la pregunta de investigación, es decir, será ésta la que "comande" o "dicte" el método de investigación o las posibles combinaciones. De esta manera, según las autoras, "...la dicotomía ya no existe. El paradigma de métodos es incluyente y se desprende naturalmente de la pregunta de investigación" (op. cit.: 15).

Así, aunque de entrada se desconoce la dicotomía "cuali-cuanti", sobre todo en su carácter de polos opuestos, señalan que se le puede emplear con fines heurísticos, o sea, para buscar caminos que lleven a su mutua consideración y utilización, ya que "entre los paradigmas cualitativos y cuantitativos hay un continuum de métodos" (Newman y Benz, 1998: 11). En este sentido se da el continuum pero también la interacción, ya que cada método retroalimenta al otro; una de las mane-

${ }^{4}$ En el mencionado artículo se da cuenta de algunas de las propuestas de los siguientes autores: Alexander, 1987; Alexander et al., 1987; Bertaux, 1988; Bertaux y Kohli, 1984; Cicourel, 1981; Collins, 1987; Cortés y García, 1993; Dávila, 1995; Fielding y Fielding, 1986; Gerstein, 1987; Knorr-Cetina, 1981; Knorr-Cetina y Cicourel, 1981; McCracken, 1988; Newman y Benz, 1998; Ortí, 1995; Saltalamacchia, 1992; y Slim y Thompson, 1993. 
ras en que se da la interacción es cuando, por ejemplo, de un análisis cualitativo surgen preguntas de investigación para un enfoque cuantitativo y viceversa. Finalmente, esta propuesta del "continuum interactivo" tiene como objetivo general que "cada enfoque añada y contribuya al conocimiento al ir construyendo sobre la información derivada desde el otro enfoque" (op. cit.: 20). Hemos retomado esta propuesta, por lo menos en un primer acercamiento, cuando intentamos establecer un diálogo entre los diferentes tipos de fuentes de datos.

Tashakkori y Teddlie (1998) constituyen otra mancuerna de autores que se ubica en la propuesta del continuum de métodos y concede suprema importancia a la pregunta de investigación frente a la elección de paradigmas o enfoques. Así, al inicio del siglo XXI la liamada "metodología mixta" retoma el esfuerzo que las ciencias sociales desarrollaron a lo largo de varios lustros para lograr una auténtica integración de enfoques, métodos y niveles de análisis; y, como nos advierten estos autores, "la preferencia por el continuum refleja la importancia de ver a los componentes del diseño de investigación como tonos del gris más que como blanco o negro" (op. cit.: 32 ).

La propuesta de la "metodología mixta" incluye la distinción entre "métodos mixtos" y "modelos mixtos". Los primeros corresponden más bien a la ya conocida y mencionada intención de combinar los enfoques cualitativos y cuantitativos; en cambio con los "modelos mixtos" se hace referencia a todo el proceso de investigación, o sea, se abarca desde el planteamiento del problema y la recolección de información hasta el análisis y los resultados, ya que comprenden todas las etapas del proceso y no sólo la combinación, por ejemplo, de fuentes de información identificadas con las dos tradiciones de las que hemos venido hablando. Existen muy pocos ejemplos de "modelos mixtos", ya que las disciplinas sociales apenas están incursionando con éxito en la implementación de los "métodos mixtos"; incluso puede decirse que en la década de los noventa persiste el problema de cómo articular los diversos elementos que contienen ambos enfoques, lo cual representa un reto permanente y vigente hasta la fecha para las ciencias sociales.

A su vez, dentro de los llamados "métodos mixtos", tanto los mencionados autores así como otros (Creswell, 1995), ofrecen la siguiente clasificación que apunta a estilos o formas diferentes de combinación de elementos:

1) Secuencial: se trata de dos etapas separadas; por ejemplo, primero se realiza un estudio cualitativo y luego uno cuantitativo, o viceversa, y finalmente se lleva a cabo su combinación. 
2) Paralelo o simultáneo: se llevan a cabo al mismo tiempo las investigaciones de corte cualitativo y las de corte cuantitativo.

3) Estatus equivalente: se utilizan ambos enfoques concediendo a cada uno el mismo peso en el entendimiento y explicación del fenómeno bajo estudio.

4) Enfoque predominante: aunque se usan ambos enfoques, desde el inicio el investigador elige a uno de ellos como predominante y el otro se considera sólo complementario.

El ejercicio que planteamos en esta oportunidad puede ubicarse en la primera clasificación (Tashakkori y Teddlie, 1998: 133), ya que inicialmente se generó la investigación de corte totalmente cualitativo sobre un grupo de mujeres de sectores medios y posteriormente se acudió a fuentes de datos estadísticamente representativas ya existentes para utilizarlas comparativamente. La manera práctica de hacer entonces la combinación "cuali-cuanti" fue identificando en cada una de las fuentes de datos las características variadas que presenta la vinculación familia-trabajo, mediante las posibles articulaciones de cuatro trayectorias vitales para el caso de las referidas mujeres de sectores medios, y se estableció así una tipología que, de inicio, surgió de la información cualitativa y luego se elaboró para la Encuesta Nacional de Empleo Urbano (Blanco y Pacheco, 2001) y para este artículo se trabajó con la Eder. Antes de desarrollar el principal objetivo de este texto, a continuación se presenta una breve reflexión en torno a uno de los elementos centrales en los análisis de corte longitudinal.

La temporalidad: una dimensión indispensable en el estudio de las trayectorias laborales

La reflexión y el manejo teórico y empírico de la dimensión temporal ha sido de gran interés para las más variadas disciplinas -desde la filosofia hasta la física pasando por la historia, por supuesto-. Sin tener la menor intención de entrar aquí a temas tan complejos como amplios y abstractos en torno a qué es el tiempo, si existe per se o si es una construcción humana o, en todo caso, sobre cómo se construye socialmente, queremos destacar, como certeramente lo hiciera desde hace años el connotado demógrafo Norman B. Ryder, que el tiempo, o sea la consideración de la dimensión temporal, tiene un lugar central en cualquier esquema y análisis demográfico, ya que lo que los 
demógrafos ven son precisamente los cambios a lo largo del tiempo (véase Ryder, 1992).

Más concretamente, y de nuevo siguiendo a Ryder, una de las preocupaciones básicas de la demografía es la medición, en este caso, del cambio de elementos específicos o de determinadas variables a lo largo del tiempo. En este sentido la edad adquiere un papel fundamental en cualquier modelo o análisis, ya que de entrada la edad individual es un índice del paso del tiempo. Por un lado, la edad se convierte en un criterio verdaderamente prominente de clasificación, ordenamiento y asignación en las sociedades y, por otro lado, el año de nacimiento (cohorte) es una especie de variable proxy que conecta al individuo y lo ubica en determinado tiempo histórico.

Esta conexión individuo-sociedad se logra más plenamente valiéndose del concepto de cohorte. En la investigación demográfica, como es ampliamente conocido, con el término cohorte se hace referencia a un grupo de personas que comparten simultáneamente una experiencia demográfica. La cohorte más clásica toma como elemento definitorio la edad, o sea, se trata de un conjunto de personas que nacieron en el mismo año calendario o pertenecen a un mismo grupo de edad. También se usa el término cohorte para referirse a otros conjuntos que experimentan o entran a un mismo evento al mismo tiempo, por ejemplo, se habla de cohortes escolares, laborales, matrimoniales, etcétera.

Uno de los supuestos o implicaciones más importantes que conlleva el término de cohorte, y que ha sido rescatado e impulsado por la sociología del envejecimiento y el enfoque del curso de vida, es que ese conjunto de personas es un grupo de edad que se mueve o transita a lo largo de la historia. Es decir, el año o periodo de nacimiento sitúa a las personas en un contexto histórico y, por lo tanto, las relaciona con una diversidad de fuerzas que operan en ese momento y que van cambiando a lo largo del tiempo (véase entre otros, Ryder, 1965; Eider y Pellerin, 1998).

Aquí cabe mencionar la existencia de una vertiente de investigación diferente pero a la vez relacionada con el análisis de cohortes que es el estudio de las generaciones. Desde hace años una variedad de autores ha utilizado el término generación como sinónimo de cohorte de nacimiento; sin embargo, existe todo un debate en torno a la conceptualización de generación como algo más amplio y complejo, que aunque está asociado es también diferente al concepto de cohorte (véase entre otros, Kertzer, 1983). En este documento utilizare- 
mos sólo el término de cohorte, aunque en otros textos sí lo hemos relacionado con el de generación (Blanco, 2001)..$^{5}$

En síntesis, una manera específica de abordar empíricamente la dimensión temporal y de dar cuenta de ciertos cambios en el tiempo es mediante el análisis de cohortes. Los estudios que optan por este camino generalmente llevan a cabo comparaciones entre cohortes diferentes; sin embargo, más recientemente se ha insistido en la pertinencia de darle seguimiento a una sola cohorte, o incluso a "subcohortes" que pueden ser distinguidas por sexo, raza, clase social, ubicación geográfica, etc. Así, como en otros muchos temas de estudio dentro de la demografía como de las ciencias sociales en general, los investigadores han pasado de una concepción lineal y homogénea del problema a la aceptación de la heterogeneidad, la discontinuidad y la contingencia (Settersten y Mayer, 1997).

En esta oportunidad hemos tomado como objeto de estudio precisamente la representación de un subconjunto poblacional muy específico: se trata de mujeres nacidas en la República Mexicana entre 1951 y 1953, que al momento de las entrevistas (1997 en el estudio cualitativo y 1998 en la Eder) vivían en contextos urbanos y contaban con un nivel de escolaridad de estudios universitarios. Es necesario especificar que aunque no hubieran terminado una licenciatura ni se hubieran titulado, el criterio de selección es que hubieran accedido a la universidad en algún momento de su vida, lo cual las ubica, de cualquier manera, como una minoría respecto al promedio nacional de escolaridad (tanto en el momento en que cursaron sus estudios universitarios como en el momento de la entrevista).

Entre las razones queijustifican el tomar como universo de estudio conjuntos que deben cubrir ciertos criterios de selección, como los anteriormente mencionados, destaca el supuesto de la no homogeneidad de las cohortes, como lo enfatizan, por ejemplo, Angela O'Rand y John Henretta (1999). Es decir, si bien el hecho de haber nacido en un mismo año sitúa en el mismo escenario histórico general a todas las personas que comparten ese evento demográfico, esas mismas circunstancias histórico-sociales inciden de manera diferente en las cohortes según,

${ }^{5}$ Una vertiente más de análisis asociada con todo lo anterior gira en torno a la famosa tríada de "efectos" mencionada en muchos estudios demográficos como "edad, periodo, cohorte"; en esta ocasión tampoco incursionaremos en la muchas veces difícil distinción entre estos tres efectos porque su tratamiento requeriría por sí mismo de un texto, aunque ciertamente su utilización es pertinente en el análisis de cohortes. 
por ejemplo, su ubicación geográfica, de clase social y (o) familia de origen, sin olvidar la siempre presente distinción entre hombres y mujeres, todo lo cual finalmente contribuye al establecimiento de diferencias y desigualdades. De esta manera, "las desigualdades dentro de las cohortes frecuentemente exceden aquellas entre cohortes y son el resultado de la interacción entre las condiciones estructurales, la acción individual y la oportunidad" (O'Rand y Henretta, 1999: 9). Una de las características distintivas del enfoque del curso de vida como perspectiva teórico-metodológica es la atención que le presta a la heterogeneidad: "empíricamente dicha heterogeneidad se observa como variaciones inter-cohortes e intra-cohortes" (George, 1996: 249).

En este complejo panorama algunos autores (Kohli, 1994; Liefbroer, 1999) destacan la importancia de los dominios escolar y laboral en la conformación del curso de vida y, por ende, en los resultados diferenciales que se observan con el correr del tiempo. Para el caso de las mujeres esto puede ser aún más claro, puesto que la segunda mitad del siglo Xx fue testigo de los grandes cambios que se experimentaron en el mundo entero, ya que más mujeres accedieron a mayores niveles de escolaridad e ingresaron paulatina pero crecientemente a los mercados de trabajo. México no es una excepción en este sentido y la variedad de estudios sobre la participación económica femenina así lo avala.

Después de esta breve reflexión metodológica, en los dos últimos apartados trataremos de articular las dos estrategias de investigación mencionadas con la idea esencial de partir del estudio cualitativo, especialmente de la construcción de una tipología que se centra en cuatro trayectorias de vida (escolar, laboral, conyugal y reproductiva) y la articulación de las mismas, para ubicar al mismo universo de estudio en las bases de datos denominadas Eder y ENEU. Sin embargo, antes de entrar al tema describiremos las características de la información que utilizaremos en este esfuerzo de análisis exploratorio.

\section{Matriz de datos}

Por lo que se refiere al recorte empírico cualitativo se partió de un estudio previo realizado por Blanco (en este mismo volumen) en el cual se da seguimiento a un grupo de mujeres consideradas de clase media, que formaban parte de un universo inicial de referencia constituido por un grupo escolar integrado por hombres y mujeres que, 
en la segunda mitad de los años sesenta, fueron compañeros de secundaria (algunos también de primaria o de preparatoria) en una escuela privada de la Ciudad de México, identificada entonces precisamente como de clase media. El primer año de secundaria tenía en 1966 dos grupos con un total de 96 alumnos, de los cuales $43 \%$ eran mujeres (datos tomados del anuario escolar de 1966). Una parte de este grupo se ha seguido reuniendo esporádicamente a lo largo de los años, y en 1996 (30 años después) hubo una reunión a la cual asistieron 19 mujeres (de las 41 que estuvieron en primero de secundaria, por lo tanto $46 \%$ de ese total) y 18 hombres. De esta manera, la simple asistencia a dicha reunión y la posibilidad de recabar una serie de datos básicos, determinaron el primer recorte en el proceso de selección de aquellas mujeres que iban a ser entrevistadas posteriormente.

Los asistentes (hombres y mujeres) contestaron un breve cuestionario que captaba datos sociodemográficos básicos, como su edad exacta, estado civil y número de hijos; en ese mismo instrumento se recabó alguna información sobre la trayectoria escolar y el tipo de trabajo que desempeñaban en ese momento. Finalmente, de las 19 mujeres que asistieron a la reunión de 1996, 13 accedieron a ser entrevistadas. ${ }^{6}$ Nacidas casi todas en la Ciudad de México y también prácticamente todas en el año de 1953 (con algunos meses de diferencia hacia el año anterior o posterior), y por lo tanto pertenecientes a una cohorte de nacimien to o generación, conforman a la vez una cohorte escolar.

La elección de las mujeres como unidad de registro y eje del análisis responde, fundamentalmente, a la conocida afirmación de que su estrecha conexión con la reproducción social le imprime una dinámica específica tanto al conjunto de la organización doméstica como a sus propias trayectorias vitales. A una muy amplia mayoría de las mujeres que en algún momento de su vida realizan un trabajo extradoméstico, sobre todo si están casadas y con hijos, se les plantea como dilema -o por lo menos como una situación un tanto conflictiva y que requiere de soluciones y estrategias- la necesidad de elegir o asignar prioridades y tiempos diferenciales a ambas esferas (la laboral y la fa-

\footnotetext{
${ }^{6}$ En el presente ejercicio no incluimos a una de las entrevistadas porque no cumple con el criterio de selección utilizado en esta ocasión de tener al menos un año de estudios universitarios (después de la secundaria cursó una carrera técnica y durante toda su trayectoria laboral se ha desempeñado como secretaria); por lo tanto, de aquí en adelante cuando se haga referencia al estudio cualitativo se estarán considerando doce mujeres.
} 
miliar-doméstica). Lo anterior, conforme lo han mostrado algunos autores (Rossi, 1985; Hagestad, 1992), se cristaliza en trayectorias vitales que contienen discontinuidades así como armonizaciones entre los diversos dominios y que terminan conformando cursos de vida femeninos más complejos o con un mayor traslape de trayectorias que su contraparte masculina.

Se fijó como delimitación temporal el periodo que va de 1950 a 1997 (año en que se realizaron las entrevistas) ${ }^{7}$ porque éste comprende una variedad de situaciones sociohistóricas que sitúan a la cohorte elegida en su inicio en un contexto de auge económico nacional, ya que estas mujeres nacen en medio del llamado "milagro mexicano" y transitan a lo largo del tiempo por diferentes momentos históricos hasta llegar a las crisis económicas de los años ochenta y noventa, proceso en el cual se presentan variadas situaciones, incluso la movilidad social descendente que ha experimentado buena parte de los sectores medios y respecto a la cual se ha investigado poco.

Por lo que se refiere al recorte empírico cuantitativo, en primer lugar cabe aclarar que aunque en esta oportunidad le hemos dado más espacio a la Eder, en realidad usaremos tanto esta encuesta como la ENEU para llevar a cabo una implementación práctica de la "metodología mixta". Es decir, en el mencionado trabajo previo (Blanco y Pacheco, 2001), se priorizó el "continuum interactivo" entre la investigación cualitativa y el seguimiento de un grupo de mujeres en la Encuesta Nacional de Empleo Urbano (ENEU), ${ }^{8}$ mientras en este nuevo ejercicio se pone el acento, dentro del enfoque de la "metodología mixta", en la realización de un ejercicio "secuencial" (como se mencionó en el primer apartado) entre los hallazgos del estudio de corte cualitativo y el seguimiento de una cohorte en la Encuesta Demográfica Retrospectiva Nacional (Eder), aplicada por el INEGI en 1998 bajo la coordinación de un grupo de investigadores de instituciones nacionales e internacionales. ${ }^{9}$ Esta es la primera encuesta biográfica mexi-

\footnotetext{
${ }^{7}$ Se llevaron a cabo entrevistas semiestructuradas pero en profundidad, que prácticamente constituyen historias de vida; todas las entrevistas se grabaron y se transcribieron integramente.

${ }^{8}$ La ENEU se realiza en México desde 1985 de manera continua y abarca en la actualidad más de $90 \%$ del conjunto urbano (100000 y más habitantes); se visita a cada familia durante cinco trimestres y después se cambia la muestra.

${ }^{9}$ Marie-Laure Coubès de El Colegio de la Frontera Norte, María Eugenia Zavala de la Universidad París-X, René Zenteno del Instituto Tecnológico de Estudios Superiores de Monterrey y Gabriel Estrella de la Universidad Autónoma de Baja California.
} 
cana representativa en el país para tres cohortes de edad (las personas nacidas entre 1933-1936, 1951-1953 y 1966-1968) que da seguimiento a la historia de vida de hombres y mujeres, rescatando sobre todo tres ámbitos: el familiar, el laboral y el migratorio. Cabe mencionar que esta encuesta permite conocer, entre otros aspectos, la trayectoria educativa de las personas encuestadas.

La Eder constituye un subconjunto (de 3200 casos) de la muestra de la Encuesta Nacional de la Dinámica Demográfica (Enadid) aplicada en 1997. La encuesta retrospectiva tiene la característica de incluir 100 casos para cada estado de la República Mexicana diferenciados por niveles de urbanización representativos para el corte de localidades de menos de 15000 habitantes y localidades de 15000 o más. Cabe aclarar que se tomaron en cuenta tres años calendario para cada cohorte porque se quería que ésta fuera lo más homogénea posible; por otro lado, todos los eventos registrados en la encuesta son aquellos que corresponden a una duración de al menos un año en la vida de los individuos.

Ahora bien, la selección en la Eder se realizó de la siguiente manera: de los 1066 hombres y mujeres encuestados, correspondientes a la cohorte 1951-1953 -años que coincide con el año de nacimiento de las mujeres entrevistadas en el estudio de corte cualitativo- se trabajó con el subgrupo de mujeres (534), y de este subgrupo se seleccionó a las que viven en localidades urbanas (254). Por último, de la población de mujeres urbanas, para el presente ejercicio se seleccionó el subconjunto de las que habían logrado cursar al menos un año de estudios universitarios (24 mujeres en la muestra, cifra que al momento de expandirse representa un total de 78 798) (gráfica 1). Este reducido número de casos está relacionado con el hecho de que para esta cohorte solamente $6.7 \%$ logró alcanzar al menos un año de universidad, con la característica de que todas se encontraban en 1998 en contextos más urbanizados. Dicha selección también está directamente relacionada con el hecho de que las mujeres del estudio cualitativo pertenecen a un sector de clase media con estudios universitarios y, de entrada, lo que se procuró fue hacer equiparables los casos seleccionados tanto en el estudio cualitativo como en las bases de datos cuantitativas.

La descripción de cómo se seleccionaron los casos en la ENEU se especifica de manera más detallada en un trabajo previo (Blanco y $\mathrm{Pa}$ checo, 2001); en esta ocasión basta señalar que el universo de estudio que seleccionamos a partir de la ENEU incluyó a las mujeres que tenían 
las siguientes características: ser residente en la Ciudad de México al momento de las entrevistas, haber alcanzado estudios universitarios y estar entre las edades de 33 a 35 años para el periodo de 1987, o entre 43 a 45 años para el periodo de 1997; la muestra final quedó conformada por 28 mujeres para 1987 y 25 para 1997.

Antes de describir las trayectorias de las encuestadas y los resultados del estudio cualitativo, vale la pena señalar ciertas ventajas analíticas que se derivan del uso secuencial de las dos bases de datos cuantitativas que hemos elegido en combinación con la información cualitativa, tal como lo contempla una de las estrategias de la llamada "metodología mixta". Así por ejemplo, con la encuesta Eder hemos podido abordar el estudio de una cohorte específica, pero dado que la representatividad es nacional, no es posible seleccionar exclusivamente a las mujeres de la Ciudad de México, aspecto que sí se pudo lograr al trabajar con la ENEU. Es decir, a partir de la Eder estaríamos hablando de mujeres urbanas que alcanzaron al menos un año de estudios universitarios, mientras con la ENEU podemos trabajar con las mujeres de la Ciudad de México que llegaron a la universidad. Por otro lado, dadas las características de la Eder, se tiene la perspectiva longitudinal, pero algunos aspectos de orden más sincrónico, como por ejemplo la carrera estudiada por las mujeres, sólo se pueden ubicar en la ENEU ya que este dato no existe en la muestra biográfica de la Eder. ${ }^{10}$

\section{Las trayectorias vitales: escolar, laboral, conyugal y reproductiva}

Este apartado está dedicado a la exposición de algunos de los principales elementos que caracterizan a las trayectorias vitales consideradas: la escolar, la laboral, la conyugal y la reproductiva. Se ha puesto un especial interés en las dos primeras haciendo que las trayectorias conyugal y reproductiva intervinieran de manera un tanto sintética (tomando en cuenta variables como el estado civil, la edad al matrimonio y el número de hijos), aunque cabe aclarar que no por ello resultan menos importantes. Posteriormente entrelazamos los hallazgos del estudio cualitativo sobre las características de las distintas trayectorias con los resultados que se obtuvieron de la selección realizada a

${ }^{10}$ Cabe aclarar que como la Eder es una submuestra de la Enadid, algunas variables de carácter sincrónico se pueden detectar en la muestra de la Enadid, pero en esta oportunidad solamente se trabajó con la muestra biográfica. 


\section{GRÁFICA 1}

Tipologías cualitativas (construcción a lo largo de la cohorte)

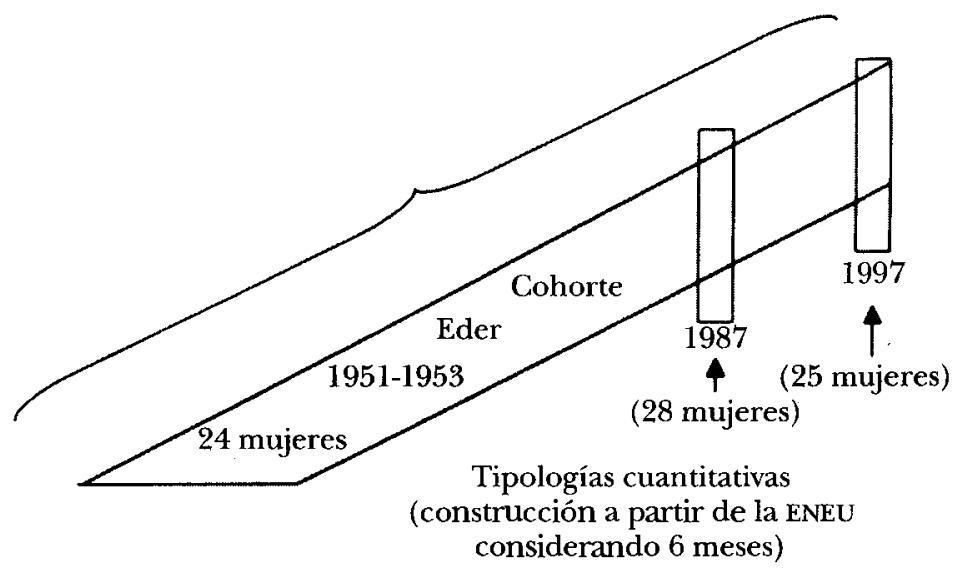

Generación 1953 (estudio cualitativo con 12 mujeres)

partir de la Eder, haciendo uso de algunos datos encontrados previamente a partir de la ENEU en caso de ser necesario. Es pertinente mencionar que como la Eder es una encuesta relativamente nueva, el análisis sobre la base de datos está realizándose actualmente y sus hallazgos aún no han sido publicados, por lo que en esta ocasión no incorporaremos los documentos inéditos existentes.

\section{Trayectoria escolar}

Es un hecho reconocido que entre las clases medias urbanas se presenta una clara tendencia hacia el acceso a la educación básica y aun a la media superior (preparatoria) (Mier y Terán y Rabell, 2001). Éste es el caso del grupo estudiado de manera cualitativa; hay que recordar que se está hablando de mujeres nacidas en la primera mitad de la década de los cincuenta; más precisamente, las 12 entrevistadas nacieron en 1953, por lo que ingresaron a la primaria en 1960, a la secundaria en 1966, a la preparatoria en 1969 y a la licenciatura en 1972. De las 12 entrevistadas, 10 terminaron una licenciatura y las otras dos la iniciaron pero la dejaron en los primeros dos años para casarse. De esta manera resalta el hecho de que la trayectoria escolar 
que presenta este grupo de mujeres de clase media corresponde no sólo a un patrón de mayor acceso a la educación por parte de las mujeres mexicanas en la segunda mitad del siglo Xx, sino a una característica propia de las clases media-media y media-alta, como es la de las mujeres profesionistas que constituyen una clara minoría respecto al contex to nacional (Mier y Terán, 1993).

Agregado a lo anterior, habría que destacar que si bien la mayoría de las entrevistadas ( 6 de 12) eligió una licenciatura dentro del área social y de humanidades, también algunas decidieron incursionar en las "ciencias duras" (como la física y las matemáticas (cuatro casos). Esta situación destaca aún más si se considera el hecho de que al momento de cursar la licenciatura -primera mitad de la década de los setenta- aproximadamente $80 \%$ de la matrícula de educación superior en México correspondía a hombres y el $20 \%$ restante de mujeres se ubicaba sobre todo en las ciencias sociales y las humanidades (Blázquez, 1992). Posteriormente, y en general no de una manera inmediata, de las 10 que terminaron una licenciatura, cinco cursaron una maestría (cuatro se titularon y una no) y de éstas, dos llegaron hasta el doctorado (una obtuvo el grado en el área de ciencias físicomatemáticas y la otra al momento de la entrevista todavía no lo obtenía en el área de ciencias sociales y humanidades).

Finalmente, del estudio cualitativo cabe destacar un elemento en particular: que para la mayoría del grupo estudiado la trayectoria escolar desde la primaria hasta el ingreso a la licenciatura se realizó de manera continua, sin interrupciones prolongadas, salvo aquellas dictadas por los propios calendarios escolares (por ejemplo, algunas esperaron algunos meses entre la terminación de la preparatoria y el ingreso a la universidad). A pesar de esta homogeneización en la secuencia de la trayectoria escolar, ya desde el inicio de la preparatoria se van desprendiendo casos que representan cursos de vida diferentes; por ejemplo, la necesidad de obtener ingresos implica para un caso el priorizar una trayectoria laboral "temprana", con respecto al resto del grupo, y la elección del matrimonio y la futura maternidad como el evento más importante en ese momento frente a las otras opciones (escolar y laboral), lleva en dos casos a interrumpir los estudios de licenciatura y a concentrarse en los ámbitos privado y doméstico.

A partir de los datos correspondientes a la Eder constatamos el carácter de grupo netamente de clase media de las mujeres elegidas de la cohorte 1951-1953, ya que como un claro indicador resalta la gran diferencia en cuanto a los años promedio de estudio que pre- 
sentan las mujeres que llegaron a obtener al menos un año de universidad frente al conjunto total de la muestra. Así, mientras para la cohorte en general los años promedio de estudio fueron sólo seis (medida longitudinalmente), el subgrupo objeto de nuestro ejercicio alcanzó un promedio de 18 años (recordemos que las 24 mujeres estudiadas a partir de la Eder representan $6.7 \%$ del total de la cohorte).

Además de lo anterior, acudir a la Eder nos permitió encontrar elementos que corroboran la diversidad en cuanto a las formas que puede adquirir la trayectoria educativa en un conjunto que presenta varias características homogéneas. Así, por ejemplo, se da la interrupción debido a diferentes causas tales como la transición al matrimonio o la maternidad (cuatro de un conjunto de nueve discontinuidades educativas), o debido quizás a necesidades económicas (las restantes cinco discontinuidades educativas, del conjunto total de 24 mujeres) para, en algunos casos, retomar la trayectoria escolar años después (en otra etapa del ciclo vital) hasta lograr algún grado de educación superior (como también ocurre en un caso en la muestra cualitativa). Finalmente, resalta otro aspecto que tiene que ver con lo anterior y está relacionado con el poco número de casos con un nivel superior a la licenciatura en la muestra de la Eder (sólo tres mujeres alcanzan el grado de maestría y ninguna el doctorado), lo cual seguramente nos está hablando de muestras diferenciales de clase media entre el estudio cualitativo y las bases de datos cuantitativas; es decir, la selección cualitativa es más bien de clases media-media y mediaalta, mientras que la muestra de la Eder (al igual que lo que ocurría con la ENEU) capta una clase media más heterogénea y menos privilegiada, que por supuesto está más en concordancia con la variedad de niveles socioeconómicos realmente existente. ${ }^{11}$

11 Sólo con la información de la ENEU pueden conocerse las características de las carreras universitarias elegidas por las mujeres. Un número reducido tenía el grado de maestría (dos en 1987 y cuatro en 1997), frente a cerca de una tercera parte que no había terminado la licenciatura. La mayor parte de ellas había estudiado una carrera en el área económico-administrativa, seguidas por aquellas cuya disciplina se ubicaba en el área social y de humanidades y el área médico-biológica. Al igual que en el estudio cualitativo, es interesante destacar que un número importante de mujeres incursionó en las denominadas "ciencias duras", pese a que cuando ingresaron a la universidad era aún poco usual. 


\section{Trayectoria laboral inicial}

En la literatura nacional e internacional sobre los mercados y la fuerza de trabajo, desde hace años se ha ido advirtiendo con mayor frecuencia la creciente y constante participación de las mujeres en la actividad económica. Las diversas investigaciones llevadas a cabo en México coinciden en señalar a la década de los ochenta como la de crecimiento más acelerado en cuanto a la participación femenina en los mercados de trabajo, lo que algunos autores atribuyen a la crisis económica que caracterizó dicho periodo. Sin embargo, ya desde la década de los setenta, cuando las mujeres entrevistadas iniciaron su trayectoria laboral, se empezó a advertir un aumento significativo de la inserción laboral femenina, asociado en este periodo a la expansión urbana y de los servicios; entre otros a los proporcionados por el Estado (Blanco, 1995).

Por lo que respecta a las características sociodemográficas de la población femenina que participaba en los mercados de trabajo, "sin lugar a dudas en los setenta, al igual que en las décadas anteriores, eran las mujeresjóvenes, no unidas y con elevados niveles de escolaridad, las que más fácilmente encontraban un lugar de trabajo por la ampliación de la urbanización, la industria y los servicios de corte capitalista" (García, 1994: 64). En esta situación se encontraba, precisamente, el grupo escolar al que pertenecen las 12 mujeres entrevistadas para el estudio cualitativo.

De éstas, 10 (recordemos que dos abandonaron la universidad para casarse y no trabajaron inmediatamente después de casadas) tuvieron su primer trabajo "estable", en promedio, a los 25 años de edad (correspondiente a 1978), una vez concluida la licenciatura. Cabe aclarar que la denominación "estable" (para hacer referencia a un empleo relacionado con sus estudios de licenciatura y de tiempo completo) se usa para oponerla a la de "eventual", que hace referencia al desempeño de trabajos ocasionales o de tiempo parcial que aproximadamente la mitad de las entrevistadas realizó alrededor de los 20 años (1973), principalmente impartiendo algún tipo de docencia en escuelas primarias, secundarias o de nivel medio superior. Esto obedeció, sobre todo, a la finalidad de contar con algún ingreso propio y no depender económicamente de los padres en forma exclusiva, o incluso para colaborar con la economía familiar.

A diferencia del grupo del estudio cualitativo, las mujeres seguidas en la Eder para la cohorte 1951-1953 en promedio emprendieron 
su primer trabajo a los 20 años (incluso cuatro inician su trabajo entre los 14 y 17 años de edad), hecho que apoya el argumento de que el grupo investigado en el estudio de corte cualitativo tiene características sociales que permiten identificarlo como de estratos socioeconómicos con mayor poder adquisitivo frente a los grupos conformados por las encuestas ENEU y Eder. Ahora bien, en este momento sólo estamos abordando el comienzo de las trayectorias laborales de las mujeres entrevistadas, por lo que más adelante, en la presentación de la tipología, podrá apreciarse cuál ha sido el desarrollo de dichas trayectorias a lo largo del tiempo.

\section{Trayectorias conyugal y reproductiva}

Empezando por la edad al matrimonio, el conjunto de las 12 entrevistadas se casó en promedio entre los 24 y los 25 años (1977-1978), con excepción de las dos que abandonaron la universidad precisamente para casarse y que, por lo tanto, lo hicieron más jóvenes (alrededor de los 20 años). En la literatura especializada sobre este tema, se ha establecido que en México, para la generación 1952-1956, la edad promedio a la primera unión fue alrededor de 19 años de edad (Zavala, 1992: 109). En un estudio más reciente, pero sólo para la generación 1955, se indica que la edad promedio a la primera unión fue de alrededor de 21 años (Quilodrán, 2001: 167). Ahora bien, a partir de los datos de la Eder se encuentra que, específicamente para la cohorte de mujeres nacidas entre 1951-1953, la edad promedio a la primera unión fue de 20.4 años, mientras las mujeres que alcanzaron al menos un año de universidad se casaron en promedio a los 22.7 años. En este sentido, la mayoría de las integrantes de la muestra cualitativa realizó un matrimonio un poco "tardío", si se compara con el promedio nacional o los resultados de la Eder, pero nuevamente más en consonancia con las especificidades de los sectores medios-medios y medios-altos.

Esto resulta aún más claro si se realizan ciertas comparaciones por nivel de escolaridad; así, nos dice Mier y Terán (1993), tomando en cuenta a las varias cohortes de mujeres que analiza un estudio realizado por ella y que abarca los años de nacimiento de 1947 a 1966: "...mientras que la mitad de las mujeres sin escolaridad se casan antes de los 18 años y a los 25 casi todas están casadas, entre las que asisten a la universidad, casi ninguna inicia una unión marital antes de los 18 años y a los 
25 años más de la mitad permanece soltera" (p. 726). De esta manera resalta la prioridad que los sectores medios-medios y medios-altos asignan a la obtención de ciertos niveles educativos por parte de las y los hijos y, por lo tanto, el "retraso" en el cumplimiento de eventos demográficos tales como el matrimonio y el nacimiento de los hijos.

En el caso de las 12 entrevistadas, casi al mismo tiempo que la mayoría estaba ingresando al mercado de trabajo de manera "estable" se dio el matrimonio, acontecimiento que no significó el abandono de la actividad laboral, como parece ser más frecuente en otros estratos socioeconómicos. No sucedió lo mismo con el ejercicio de la maternidad, ya que el hecho de tener hijos sí influyó en la decisión de dejar de trabajar durante algún tiempo. Así, la mayoría de las que trabajaban dejaron de hacerlo durante un tiempo (por ejemplo, un año) para dedicarse de manera exclusiva al cuidado del recién nacido; no siempre ocurrió lo mismo, pues algunas sólo se tomaron el tiempo estipulado por ley y, en todo caso, trataron de alargar el periodo sin trabajar, adicionando a la incapacidad, por ejemplo, las vacaciones.

Por otro lado, el nacimiento del primer hijo ocurrió, en promedio, unos dos años después del matrimonio. Es decir, estas joóvenes mujeres profesionistas de finales de los años setenta contaban con la información necesaria para controlar su fecundidad y eligieron cuándo y cuántos hijos tener. Prueba de ello es que, en promedio, tuvieron dos hijos, ${ }^{12}$ cuando la cifra nacional, por ejemplo para 1980 , era de 4.4 hijos por mujer, considerando que México ya se encontraba en pleno descenso de sus tasas de fecundidad (Zavala, 1992: 93-94); este último resultado coincide con el de la cohorte correspondiente a los años 1951-1953 de la Eder.

Un primer aspecto por resaltar a partir de los resultados de la Eder es la diferencia significativa por estado civil entre las mujeres que alcanzaron al menos un año de estudios universitarios y las que no lo lograron; así, mientras estas últimas en su mayoría se encontraban casadas o unidas $(81.4 \%)$, de las primeras se ubicaba en esta categoría sólo $41.5 \%$; además, el estado conyugal de separación o divorcio tiene mayor peso para las mujeres profesionistas $(43.3 \%$ para las

${ }^{12}$ De las 12 entrevistadas, sólo una tiene tres hijos, precisamente la única que ha sido ama de casa en exclusividad; de las que tienen dos hijos (la mayoría) algunas manifestaron que tal vez hubieran deseado tener otro; y las casadas sin hijos (dos casos) afirmaron que dicha situación no se debía a una decisión deliberada sino a problemas de salud. 
que cursaron algún año de universidad frente a $8.3 \%$ del grupo restante). Este último resultado puede interpretarse desde diferentes vertientes, una de ellas es que las mujeres profesionistas cuentan con más recursos económicos para poder separarse y, así, vivir de manera independiente cuando la relación de pareja no funciona.

A diferencia de los resultados obtenidos en el estudio cualitativo, a partir de los datos de la Eder se observa que un número reducido de mujeres (cuatro de un conjunto de 24) presenta la característica de ingresar al mercado de trabajo casi al mismo tiempo que se une. A la vez, las mujeres profesionistas de la EDER que salen del mercado de trabajo al momento de unirse o tener el primer hijo son también un número reducido (dos en el primer caso y tres en el segundo). ${ }^{13}$ Con la información de la ENEU no parecía existir una diferencia tan fuerte, lo cual podría atribuirse a que la utilización de datos realmente longitudinales hace posible un análisis más amplio que logra captar una mayor diversidad de las formas en que se entrelazan sus diferentes trayectorias vitales. Al respecto, algunos autores han hablado desde hace tiempo de la simultaneidad de roles de las mujeres en contraposición con las secuencias lineales y ordenadas de episodios o transiciones, lo cual no quiere decir que hayan desaparecido sino que “...los roles a través de los dominios se vuelven cada vez más interdependientes a lo largo del tiempo" (O'Rand y Henretta, 1999: 85). La tipología que presentamos en el siguiente apartado es precisamente una esquematización de la interrelación de roles y trayectorias vitales tomando en cuenta la dimensión diacrónica.

Finalmente, por lo que toca al ámbito de la fecundidad, con el ejercicio cuantitativo se constata la baja fecundidad de las mujeres de clase media (1.6 hijos para la cohorte de la Eder) y aparece como novedoso el hecho de que el grupo de estudio comprende un número significativo de mujeres solteras (cuatro) o sin hijos (seis). A partir de la información de la ENEU también se observa una proporción considerable de mujeres solteras (ocho de 28 en 1987 y seis de 25 en 1997). Este aspecto lleva a la reflexión y, entre otras inferencias, puede pensarse que las mujeres universitarias no sólo posponen la edad en que se casan, sino que también pueden optar por el "no matrimonio".

\footnotetext{
${ }^{13}$ Cabe aclarar que el fenómeno base para el cálculo de la muestra de la Eder fue la migración; esto podría llevar a pensar en que probablemente por ello se privilegien más los aspectos laborales que las dinámicas reproductivas.
} 
En esta primera presentación de los principales elementos que caracterizan las cuatro trayectorias vitales elegidas, el objetivo ha sido ver las similitudes y diferencias del universo cuantitativo respecto a la información derivada del estudio cualitativo, teniendo en cuenta la dificultad que representa conjuntar fuentes de datos con características muy diferentes, así como cortes diacrónicos y sincrónicos, de ahí que tal vez la exposición resulte un tanto descriptiva y lineal; por ello a continuación se presenta una tipología que da cuenta de las posibles articulaciones de las trayectorias vitales hasta aquí analizadas.

\section{Una propuesta tipológica}

En el trabajo que dio origen a este ejercicio, o sea el estudio cualitativo, una vez que se expuso el perfil general del grupo bajo estudio y su ubicación dentro del contexto más amplio al cual pertenece, o sea su cohorte de nacimiento o generación, fue preciso hilar más fino y entrar a la diversidad que casi siempre contiene la homogeneidad que suele percibirse en un análisis más agregado. Así, se trató de dar cuenta de las diferencias y las semejanzas valiéndose de la construcción de una tipología que incorporó el entrelazamiento de las cuatro trayectorias vitales consideradas, pero donde la trayectoria laboral se tomó como eje del análisis.

Así, en el estudio cualitativo, punto de partida de este trabajo, se situó a las entrevistadas en dos polos que, al parecer, están presentes en cualquier grupo socioeconómico y ocupación específica que desempeñan las mujeres: el no haber trabajado "nunca", ni antes ni después de casarse, y el haber trabajado "siempre", antes y después de casarse e incluso sin abandonar el trabajo en los periodos en que se han tenido hijos (con excepción de los tiempos de incapacidad que marca la ley).

Se incorporaron las trayectorias conyugal y reproductiva, precisamente por su estrecha interrelación, aunque se hizo de una manera un tanto puntual, considerando los parámetros fundamentales y sin abordar los significados que las propias mujeres atribuyen al entrelazamiento de sus trayectorias vitales. ${ }^{14}$ La investigación de corte cuali-

${ }^{14}$ En el estudio cualitativo sí se incluyeron aspectos como las percepciones de las mujeres respecto a cada una de sus trayectorias vitales y al entrelazamiento de éstas, así como la priorización o combinación que han hecho de cada dominio, pero en los ejer- 
tativo destacó un punto central: tomando en cuenta que la mayoría de las mujeres entrevistadas había desempeñado un trabajo remunerado en algún momento de su vida, tan to estando solteras como casadas, se trató de ver las diferentes modalidades que había adquirido la diada familia-trabajo, es decir, cómo habían ejercitado o desarrollado estas mujeres dicha vinculación.

Para dar cuenta de tales modalidades se construyeron cuatro tipos que toman en consideración toda la trayectoria laboral de las mujeres a lo largo del periodo estudiado (aproximadamente 20 años) y donde el factor definitorio fue la importancia o preeminencia que le habían atribuido a la esfera laboral o a la familiar-doméstica. Así, a pesar de que en tres de los cuatro tipos había estado presente a lo largo de los años la vinculación familia-trabajo, se reitera que precisamente se trató de hilar más fino y ver la diversidad y los matices que cada tipo conllevaba, con lo cual se pretendía dar cuenta de las diferentes maneras en que se vinculan las mujeres a lo largo de su vida con el trabajo extradoméstico, rechazando así la idea de la homogeneidad por clase y por etapa del ciclo vital.

Así, en primer lugar se distinguieron dos grandes vertientes, los grupos $\mathrm{A}$ y $\mathrm{B}^{15}$ y en segundo lugar dos tipos de trayectorias contenidas en los dos grupos principales:

Grupo A (1): incluye a aquellas mujeres que han priorizado la vida familiar frente al mundo laboral.

A.1. (1.1.) Nunca se ha trabajado de manera extradoméstica o el periodo dedicado a la esfera laboral ha sido mucho menor en años que el dedicado al ámbito familiar (dos casos).

A.2. (1.2.) Es clara la prioridad que se le ha asignado a la vida familiar, pero a pesar de ello a lo largo de los años se han realizado actividades laborales eventuales en tiempo parcial, esporádicas e incluso filantrópicas; además el ingreso que generan estas actividades siempre se ha considerado complementario de la economía familiar, o sea del sueldo del marido (cuatro casos).

Grupo B (2): incluye a aquellas mujeres que han tratado más activamente de vincular familia y trabajo.

cicios exploratorios de la "metodología mixta" no se ha incorporado esta información porque requeriría de otro tipo de tratamiento y, además, porque uno de los objetivos centrales de estos ejercicios ha sido, precisamente, el ensayo operativo del uso combinado de fuentes de datos que presentan características muy diferentes.

${ }^{15}$ Para facilitar la lectura de los diagramas se ha denotado con letras la tipología aplicada en el estudio cualitativo y con números su correlato cuantitativo. 
B. 1. (2.1.) Es la trayectoria que presenta la mayor continuidad; se ha trabajado desde antes de casarse y la maternidad no ha implicado interrupciones prolongadas o renuncia al empleo (en dos casos, ya que en el otro no hay hijos); además siempre se ha trabajado en el mismo tipo de ocupación o incluso en la misma institución (en los tres casos).

B.2. (2.2) A pesar de que hay discontinuidad, por haber cambiado repetidas veces de lugar de trabajo o de tipo de ocupación (por ejemplo de la academia al sector público o a la iniciativa privada y viceversa, desempeñando diferentes tipos de puestos y actividades), y por haber permanecido algunos periodos más o menos largos sin trabajar (dos a tres años) debido fundamentalmente a la maternidad, se manifiesta el interés, y a veces también la necesidad económica (por ejemplo, en el caso de las divorciadas) de tener un empleo, ingresos y también un desarrollo profesional (tres casos).

Así, los cuatro tipos dieron cuenta del entrelazamiento de trayectorias vitales y no sólo se adaptan al conjunto de las 12 entrevistadas, sino que en realidad son aplicables a todo el grupo escolar original de 41 mujeres. Sin embargo siempre habrá casos aislados o "especiales" que pueden representar aquella famosa "excepción que confirma la regla". Por ejemplo, en esta generación escolar un caso derivó en una franca movilidad social descendente, ya que presentó a lo largo de los años una trayectoria laboral "caótica", aparejada a una trayectoria conyugal y reproductiva igualmente "inusual" respecto al resto del grupo (tres matrimonios, dos de los cuales terminaron en viudez y cuatro hijos producto de esas tres uniones).$^{16}$

Ahora bien, por lo que respecta a la construcción de una tipología a partir de la base de datos de la Eder buscamos, en primer lugar, ubicar la proporción de mujeres que nunca había trabajado (subgrupo A.1 de la tipología cualitativa). Al respecto cabe mencionar que de la cohorte de las mujeres nacidas entre 1951 y 1953 nunca había tra-

${ }^{16}$ Se han documentado casos similares de mujeres pertenecientes a familias de origen de clase media, con una infancia y una adolescencia igualmente "clasemediera", que después de casarse iniciaron un largo proceso de movilidad social descendente, entre otras razones debido a la asunción de la jefatura del hogar, hasta llegar a puntos verdaderamente "extremos" o crínieos (por ajemplo, desempeñar trabajos manuales o en el sector informal o "colocar" a algún hijo en otra familia ante la imposibilidad de mantenerlo), adoptando así verdaderas estrategias de sobrevivencia anteriormente sólo asociadas a los sectores urbano-populares o a los grupos sociales más desfavorecidos (Blanco, 1997). 
bajado 35.7\%; no obstante, esta característica correspondió al grupo que no había alcanzado al menos un año de universidad. En consecuencia, el grupo de estudio de 24 mujeres seleccionado a partir de la Eder resultó ser aquel que comprende exclusivamente a las que alguna vez en su vida habían trabajado, quedando fuera de consideración el tipo 1.1., ya que remite a la situación de no haber realizado nunca un trabajo extradoméstico, o que el tiempo dedicado a éste fue considerablemente menor que el dedicado al hogar. Al respecto nos interesa resaltar una ventaja al enmarcar una investigación en la llamada "metodología mixta": utilizar fuentes de datos (ya sea simultánea o secuencialmente) con caracteristicas diferentes permite no sólo obtener información complementaria, pues lo que no está en una fuente se puede encontrar en la otra, sino también obtener pistas sobre nuevas líneas de investigación al hallar datos en las diferentes fuentes que pueden ser incluso contradictorios. Este es el caso de la ENEU, desde donde fue posible aproximarnos al grupo de mujeres que nunca habían trabajado, cuya caracterización mostró que nunca habían desempeñado una labor remunerada cinco de las 28 seleccionadas en 1987 y sólo una de las 25 seleccionadas en 1997. La reducción del número de mujeres que no había trabajado nos llevó -en el documento previo- a plantear una hipótesis sobre la necesidad que se advierte en las mujeres de clase media de incorporarse al mercado de trabajo, ya fuera porque habían finalizado su periodo reproductivo, porque buscaban un proyecto personal o por necesidades económicas (de hecho, mientras en 1987 las mujeres respondían nunca haber buscado trabajo porque no tenían necesidad, en 1997 esta era una respuesta ausente).

Volviendo a la aproximación de la tipología por medio de la Eder, un primer subgrupo correspondería a las mujeres que han privilegiado su vida familiar (cinco en la muestra de la cohorte de la Eder, que representan $8.4 \%$ del universo de estudio en la muestra expandida), el cual denominamos 1.2 (véase el diagrama). Esta agrupación sería una aproximación al subgrupo A.2 de la tipología cualitativa, que incluye a las mujeres con mayor número de hijos y, que además suspendieron su trayectoria laboral al unirse o tener hijos; asimismo la duración de sus trayectorias laborales (entre 9 y 20 años) es menor que la de los subgrupos que revisaremos a continuación. Un matiz de este subgrupo es que dos de las mujeres privilegiaron su vida familiar en etapas iniciales o intermedias del ciclo de vida, pero al momento de la entrevista se encontraban separadas y trabajando. Ca- 
be mencionar que en el interior de un universo aparentemente homogéneo, como es este primer subgrupo, es posible reconocer cierta diversidad interna; incluso algunas trayectorias no siguen el modelo "tradicional" conyugal, sino que presentan segundas y terceras nupcias y, también, periodos prolongados de separación.

Un segundo subgrupo, que denominamos 2.1 (véase el diagrama), sería la aproximación al subgrupo B.1 de la tipología cualitativa y representaría el opuesto de las mujeres que nunca trabajaron, pues corresponde a aquellas que presentan trayectorias laborales continuas (13 en la muestra de la cohorte de la Eder, que representan $64.1 \%$ del total de mujeres que alcanzaron al menos un año de universidad con la muestra expandida). En este subgrupo hay casadas, separadas o divorciadas y solteras, por ello se podría decir que se aprecia cierta heterogeneidad dentro de una característica común (no obstante, la mayor proporción corresponde al grupo de casadas o unidas); también cabe mencionar que a este subgrupo corresponde el mayor número de mujeres que no tienen hijos. Destaca el hecho de que el número de años trabajados varíe entre 23 y 34 de actividad económica continua, siendo éste el periodo más largo del conjunto.

Un tercer subgrupo, que denominamos 2.2 (véase el diagrama), corresponde a las mujeres que aunque han trabajado de manera intermitente han privilegiado las labores extradomésticas frente a las domésticas (cuatro mujeres en la muestra de la cohorte de la Eder, las cuales representan $18 \%$ del universo de quienes llegaron a la universidad con la muestra expandida). Ésta sería una aproximación del subgrupo B.2 de la tipología cualitativa. Cabe mencionar que este subgrupo, al igual que el anterior, también incluye solteras, e incluso el número de hijos es muy similar en ambos casos, pero una de las diferencias frente al grupo anterior radica en el número de años trabajados, ya que el rango se encuentra en el intervalo de 16 a 26 , con discontinuidades laborales de menos de cuatro años y con la característica de que la edad de ingreso al primer trabajo es mayor que en el caso previo. Por su parte, en la tipología de la ENEU este subgrupo dedicaba más horas al trabajo extradoméstico que al doméstico, aspecto que no es posible conocer con los datos de la Eder.

Por último, a dos de las 24 mujeres de la muestra (9.5\% con expansión) no fue posible ubicarlas dentro de la tipología (véase el diagrama), dado que son solteras y sin hijos; en consecuencia no es posible analizar el entrelazamiento de la diada familia-trabajo desde la perspectiva que hemos seguido en este ejercicio, o requeriríamos ma- 
yor información para saber, por ejemplo, si ellas priorizaron el ámbito familiar en relación con su familia de origen, dedicándose, como a veces sucede, al cuidado permanente de los padres, que es precisamente la situación del caso del estudio cualitativo que no tomamos en cuenta en esta ocasión por no haber llegado a la universidad. También nos remite al aspecto metodológico de la "activación en paralelo", de la que hablan algunos de los autores presentados en el primer apartado, ya que en cierta forma las tres fuentes de datos utilizadas presentan casos de mujeres solteras, pero en algunas este fenómeno aparece con más peso que en las otras, y aunque esto seguramente tiene que ver con el aspecto de la representatividad de cada una de las fuentes, el hecho es que nos lleva a la futura consideración de una situación familiar y laboral muy poco estudiada, así como, más específicamente, a la creciente variabilidad en los tipos existentes de trayectorias laborales.

\section{Consideraciones finales}

En el presente artículo, como se advirtió en la introducción, se ha pretendido hacer un ejercicio en torno al problema metodológico que representa la utilización simultánea de fuentes de datos que provienen de dos estrategias de investigación que durante años se han considerado opuestas. Es necesario reiterar que, en nuestro caso, se trata de las primeras aproximaciones, por lo que, como muchas veces sucede en otros temas de investigación relativamente poco explorados, el resultado es, de entrada, mas bien descriptivo.

Recordemos que se partió de un estudio cualitativo, y que retomando de la perspectiva del curso de vida la propuesta del entrelazamiento y el traslape de las trayectorias vitales, se procedió a examinar cómo se interrelacionan y coexisten cuatro de las principales trayectorias vitales femeninas, tomando como eje del análisis a la trayectoria laboral. A la pregunta de ¿qué tipo de combinaciones se dan en la interrelación familia-trabajo en mujeres de sectores medios?, la búsqueda de respuesta nos llevó a la elaboración de una tipología que da cuenta de la heterogeneidad y diversidad en el interior de un universo que es de entrada, bastante homogéneo.

Lo anterior tiene como trasfondo el señalamiento que se hace en la mayoría de los estudios sobre trayectorias laborales donde se refrenda el hallazgo de que las trayectorias masculinas tienden a ser 
mucho más continuas que las femeninas, más bien caracterizadas por la discontinuidad en el largo plazo, y la intermitencia en el corto. Partiendo de ello, en esta presentación nos hemos propuesto dar cuenta de la heterogeneidad interna que contienen las trayectorias femeninas incluso en universos que, como se ha reiterado, de entrada son bastante homogéneos, sobre todo si se les comparara con subpoblaciones muy diferentes, como por ejemplo los estratos socioeconómicos depauperados. Así, la búsqueda de diferencias y matices resultó un objetivo común para los dos tipos de fuentes de datos utilizados; de hecho consideramos que el haber conjuntado un recorte de tipo cuantitativo con los resultados previos del estudio cualitativo fue precisamente lo que hizo posible enriquecer a la vez que reforzar la propuesta de la existencia de la diversidad dentro de la homogeneidad.

Como se ha observado repetidas veces, el ánimo inicial de nuestra propuesta se centró en la dimensión metodológica, y más específicamente en la parte operativa de cómo utilizar secuencialmente, a la vez que de manera combinada, fuentes de datos que casi inevitablemente siguen siendo clasificadas como polos, en el mejor de los casos, de un continuum. Después de haber confrontado diferentes tipos de fuentes de información, llama la atención que el conjunto de casos del estudio cualitativo refleja o contiene, en efecto, las características generales que presentan universos similares extraídos de bases de datos estadísticamente representativas. Se puede decir que algunas variables que conforman el perfil sociodemográfico del conjunto de los casos cualitativos encuentran su correlato en el comportamiento similar de las mismas variables de las submuestras de la ENEU y de la Eder. Además, no sólo las variables aisladas son similares sino incluso ha sido posible construir una tipología parecida con las poblaciones de ambas fuentes, lo cual refuerza los supuestos de la llamada "metodología mixta”.

Por último, una vertiente más de análisis, surgida de esta aproximación al manejo conjunto de fuentes de datos completamente diferentes, y que habrá que explotar en el futuro, nos remite al manejo, también simultáneo, de las dimensiones diacrónica y sincrónica y a la importancia del contexto sociohistórico, como lo apunta, por ejemplo, el seguimiento de una cohorte ficticia en dos puntos en el tiempo que abarcan 10 años, en el caso de la ENEU, y a la perspectiva longitudinal que permite la Eder.

Todo lo anterior reafirma, entre otras cosas, las dificultades prácticas que señala la mayoría de los especialistas que participan en la 
discusión metodológica y epistemológica sobre la oposición, vinculación e incluso el continuum de lo "macro-micro" y lo "cuanti-cuali", pero que, a final de cuentas, tampoco se ha logrado resolver plenamente de manera teórico-metodológica. A pesar de todo, o precisamente por esto mismo, la necesidad de la articulación de niveles de análisis, de métodos y de fuentes de datos sigue vigente, por lo que habrán de encarar ese reto tanto las ciencias sociales en general como la sociodemografia en particular. 


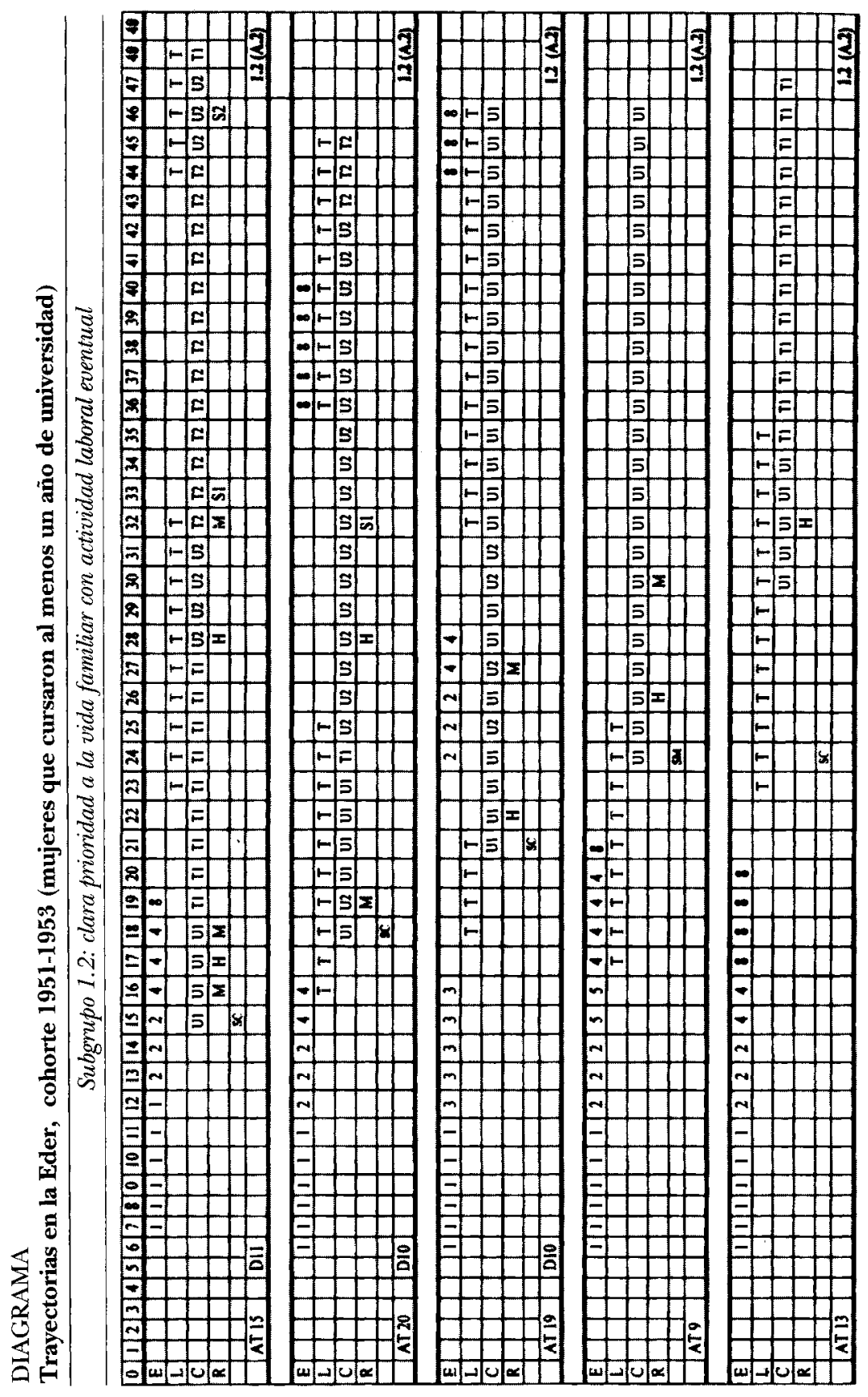




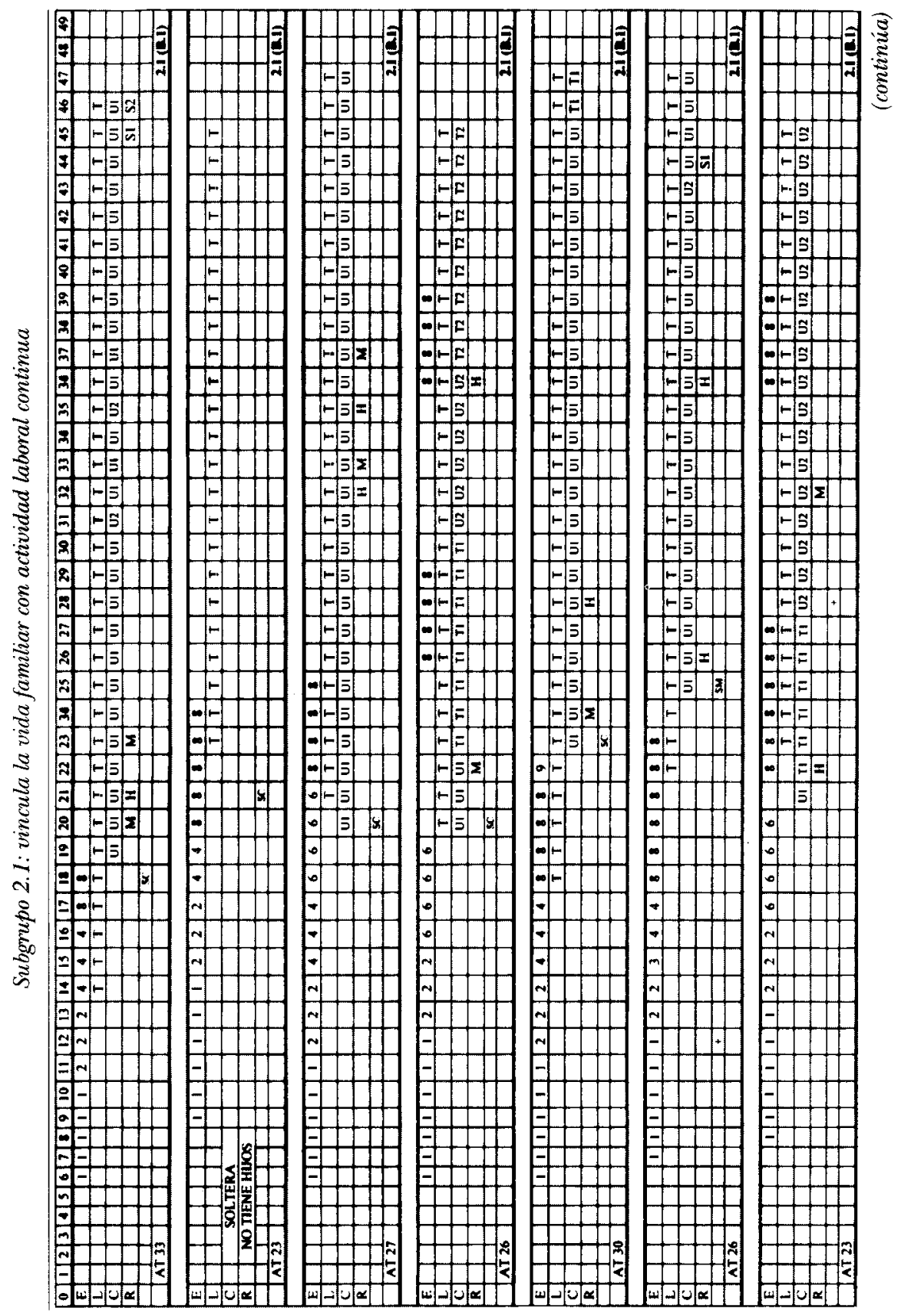




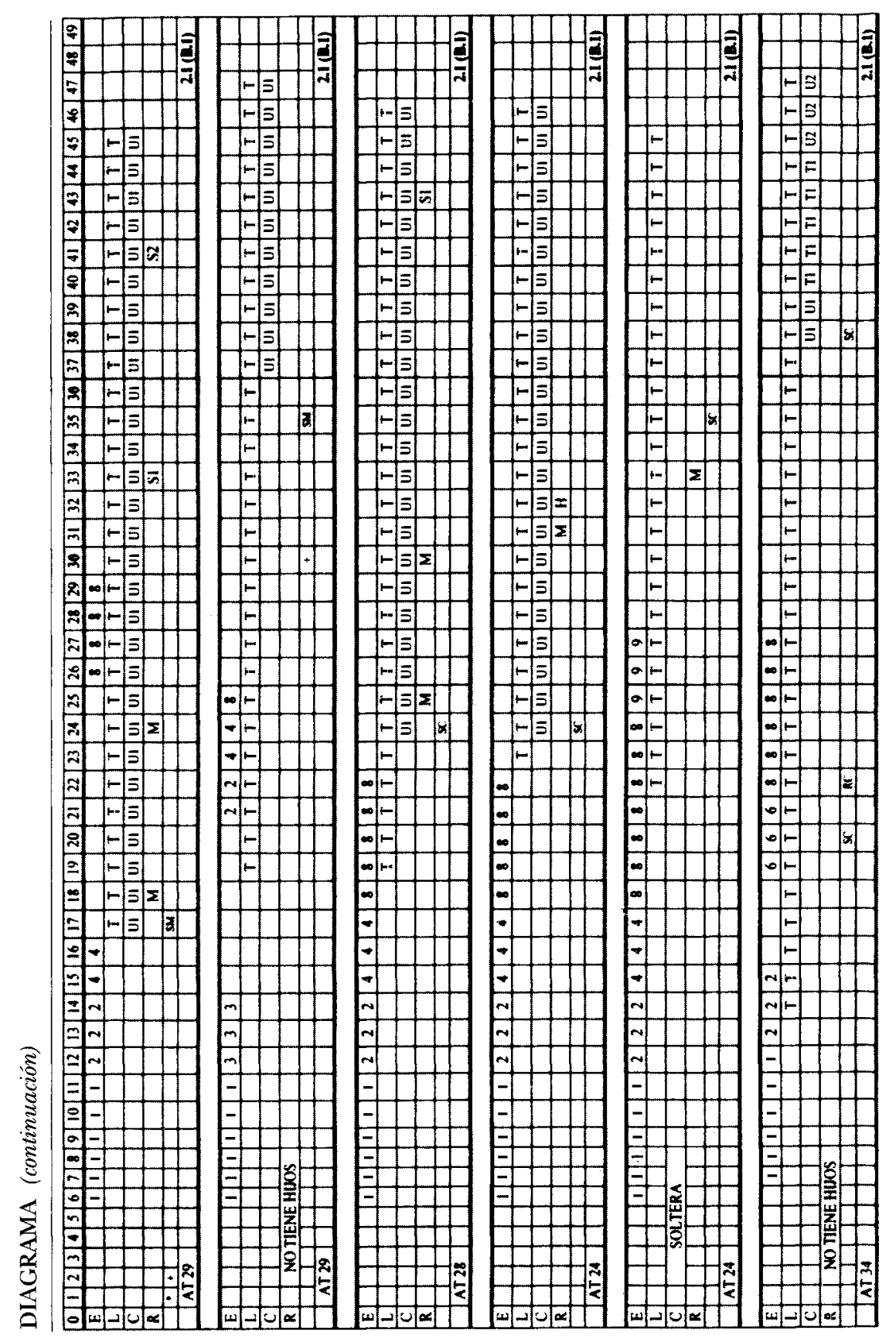




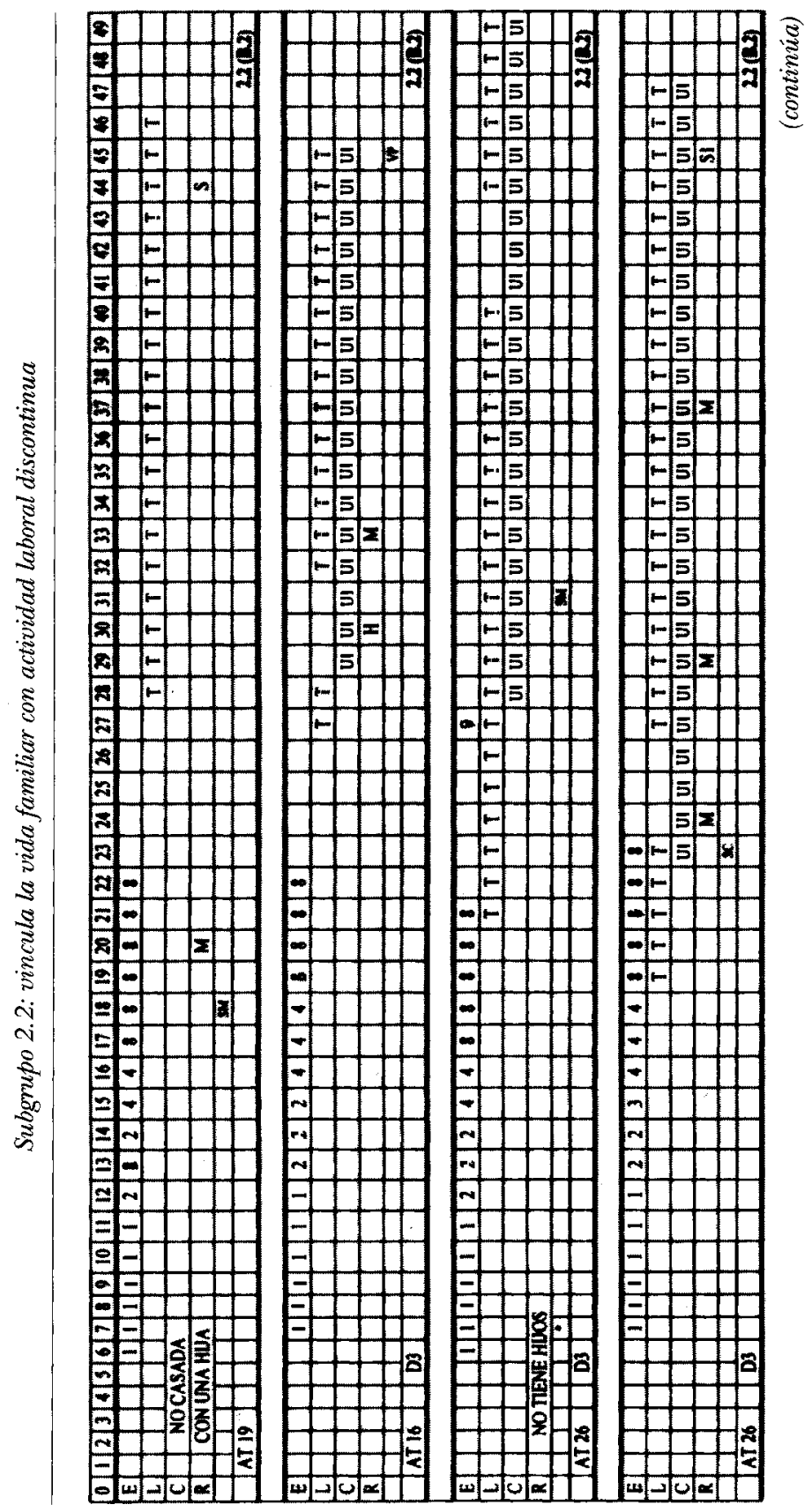




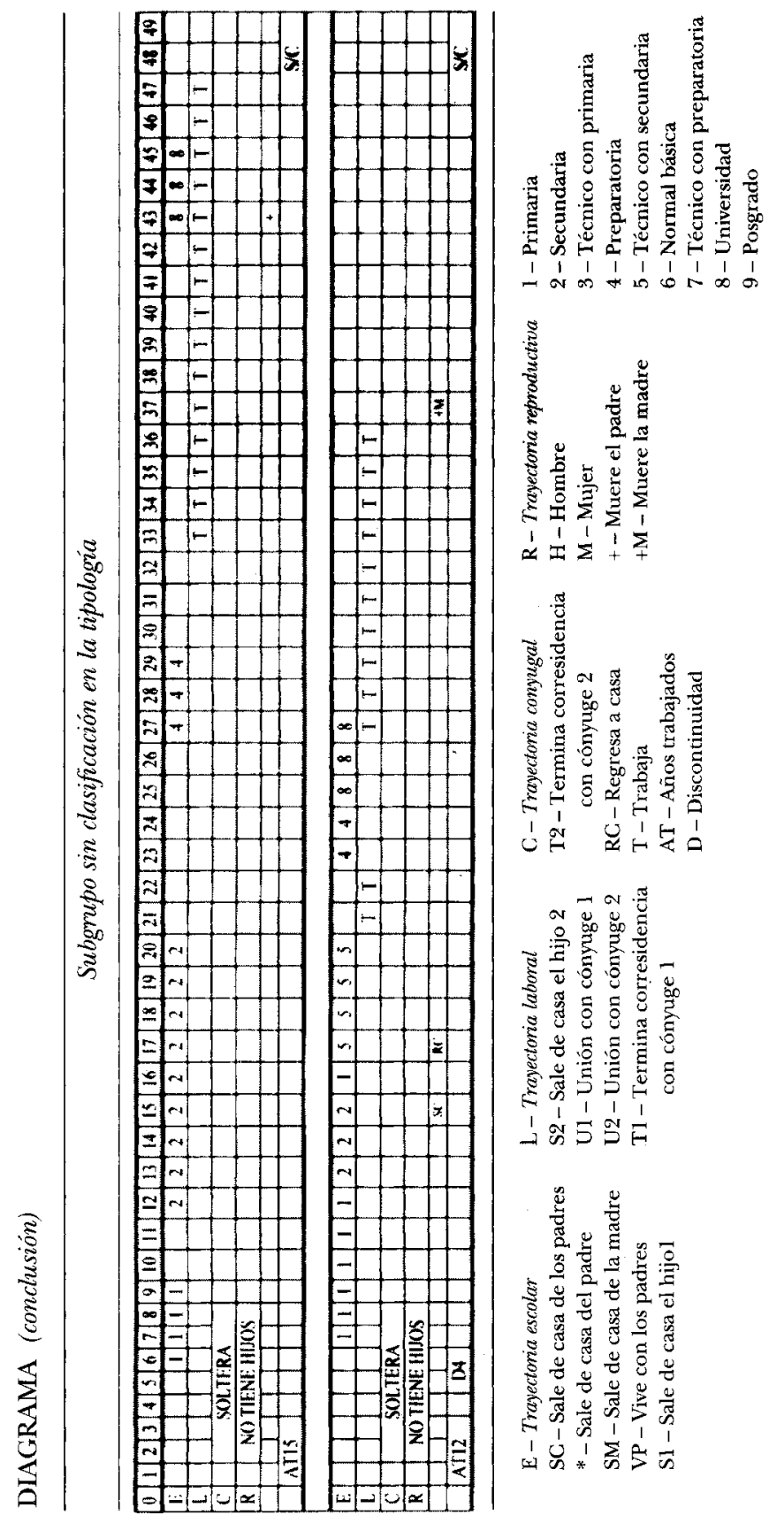




\section{Bibliografía}

Alexander, J. (1987), "Action and Its Environments", The Micro-Macro Link, Berkeley, University of California Press, pp. 289-318.

_ et al. (1987), The Micro-Macro Link, Berkeley University of California Press.

Bertaux, D. (1988), "El enfoque biográfico: su validez metodológica, sus potencialidades", Historia oral e historias de vida, Costa Rica, Flacso, pp. 55-80 (Cuadernos de Ciencias Sociales, 18).

- y M. Kholi (1984), "The Life Story Approach: A Continental View", Annual Review of Sociology, p. 215-237.

Blanco, M. (2002), "Trabajo y familia: entrelazamiento de trayectorias vitales", Estudios Demográficos y Urbanos, vol. 17, núm. 3 (51) (en este volumen).

(2001), "Trayectorias laborales y cambio generacional: mujeres de sectores medios en la Ciudad de México", Revista Mexicana de Sociologia, vol. 63, núm. 2, pp. 91-111.

- (1997), "Respuestas ante el deterioro de la calidad de vida de familias de mujeresijefas de clase media en la Ciudad de México: un estudio de caso", Memorias de la TV Conferencia Iberoamericana Sobre Familia, vol. 6, Familia, trabajo y género, Cartagena de Indias, Colombia, Universidad Externado de Colombia, pp. 93-99.

- (1995), Empleo público en la administración central mexicana. Evolución y tendencias (1920-1988), México, CIESAS (Colección Miguel Othón de Mendizábal).

- y E. Pacheco (2001), "Trayectorias laborales en la Ciudad de México: un acercamiento exploratorio a la articulación de las perspectivas cualitativa y cuantitativa", Revista Latinoamericana de Estudios del Trabajo, año 7, núm 13, pp. 105-137.

Blázquez, N. (1992), "Incorporación de la mujer a la ciencia a comienzos de los noventa", en M. L. Tarrés (comp.), La voluntad de ser. Mujeres en los noventa, México, PIEM, El Colegio de México, pp. 195-210.

Cicourel, A. (1981), "Notes on the Integration of Micro and Macro-Levels of Analysis", en K. Knorr-Cetina y A. Cicourel (eds.), Advances in Social Theory and Methodology. Toward an Integration of Micro and Macro-Sociologies, Boston, Routledge and Kegan, pp. 51-80.

Collins, R. (1987), "Interaction Ritual Chains, Power and Property: The Micro-Macro Connection as an Empirically Based Theoretical Problem", The Micro-Macro Link, Berkeley, University of California Press, pp. 193206.

Cortés, F. y R. Garcia (1993), "Muestreo estadístico, bases de datos y sistemas complejos", en I. Méndez y P. González Casanova (coords.), Matemáticas y ciencias sociales, México, CIIH, UNAM/Porrúa.

Creswell, J. W. (1995), Research Design: Qualitative and Quantitative Approaches, Thousand Oaks, Calif., Sage. 
Dávila, A. (1995), "Las perspectivas metodológicas cualitativa y cuantitativa en las ciencias sociales: debate teórico e implicaciones praxeológicas", en J. M. Delgado y J. Gutiérrez (coords.), Métodos y técnicas cualitativas de investigación en ciencias sociales, Madrid, Síntesis Psicología, pp. 69-83.

Elder, G. y L. Pellerin (1998), "Linking History and Human Lives", en J. Giele y G. Elder (eds.), Methods of Life Course Research, Qualitative and Quantitative Approaches, Thousand Oaks, Calif., Sage.

Fielding, N. y J. Fielding (1986), Linking Data, Newbury Park, Calif., Sage (Qualitative Research Methods, vol. 4).

García, B. (1994), "Determinantes de la oferta de mano de obra en México", México, Secretaría del Trabajo y Previsión Social (Cuadernos de Trabajo, 6).

George, L. K. (1996), "Missing Links: The Case for a Social Psychology of the Life Course", The Gerontologist, vol. 36, núm. 2, pp. 248-255.

Gerstein, D. (1987), "To Unpack Micro and Macro: Link Small with Large and Part with Whole", The Micro-Macro Link, Berkeley, University of California Press, pp. 86-116.

Hagestad, G. (1992), "Assigning Rights and Duties: Age, Duration, and Gender in Social Institutions", en W. Heinz (ed.), Institutions and Gatekeeping in the Life Course, vol. 3, Weinheim, Deutscher Studien Verlag, pp. 261-279.

Hentschel, J. (1999), "Contextuality and Data Collection Methods: A Framework and Application to Health Service Utilisation", The Journal of Development Studies, vol. 35, Londres, http://www.umi.com/proquest, pp. 64-94.

Kertzer, D. (1983), "Generations as a Sociological Problem", Annual Review of Sociology, vol. 9, pp. 125-149.

Knorr-Cetina, K. (1981), "The Micro-Sociological Challenge of Macro-Sociology: Towards a Reconstruction of Social Theory and Methodology", en K. Knorr-Cetina y A. Gicourel, Advances in Social Theory and Methodology. Toward an integration of Micro and Macro-Sociologies, Boston, Routledge and Kegan, pp. 1-47.

y A. Cicourel (1981), Advances in Social Theory and Methodology. Toward an Integration of Micro and Macro-Sociologies, Boston, Routledge and Kegan.

Kohli, M. (1994), "Work and Retirement: A Comparative Perspective", en M. W. Riley, R. L. Kahn y A. Foner (eds.), Age and Structural Lag, Nueva York, John Wiley and Sons, pp. 80-106.

Liefbroer, A. C. (1999), "From Youth to Adulthood: Understanding Changing Patterns of Family Formation from a Life Course Perspective", en L. J. G. van Wissen y P. A. Dykstra (eds.), Population Issues. An Interdisciplinary Focus, Nueva York, Kluwer Academic/Plenum, pp. 53-85.

McGracken, G. (1988), The Long Interview, Newbury Park, Calif., Sage.

Mier y Terán, M. (1993), "Trayectoria de vida de las mujeres jóvenes en México", TV Conferencia Latinoamericana de Población. La transición demográfica en América Latina y el Caribe, vol. 2, México, INEGI/ISSUNAM, pp. 721-745. 
C. Rabell (2001), "Condiciones de vida de los niños en México, 19601995; el entorno familiar, la escolaridad y el trabajo", La población de México: tendencias sociodemográficas y perspectivas hacia el siglo XXX, México, Consejo Nacional de Población/Fondo de Cultura Económica, pp. 725-758.

Newman, I. y C. Benz (1998), Qualitative-Quantitative Research Methodology. Exploring the Interactive Continuum, Southern Illinois University Press.

O'Rand, A. y J. Henretta (1999), Age and Inequality, Diverse Pathways Through Later Life, Boulder, Westview Press.

Orti, A. (1995), "La confrontación de modelos y niveles epistemológicos en la génesis e historia de la investigación social", en J. M. Delgado y J. Gutiérrez (eds.), Métodos y técnicas cualitativas de investigación en ciencias sociales, Madrid, Síntesis Psicología, pp. 85-95.

Quilodrán, J. (2001), Un siglo de matrimonio en México, México, El Colegio de México.

Rossi, A. (ed.) (1985), Gender And The Life Course, Nueva York, Aldine.

Ryder, N. (1992), "The Centrality of Time in the Study of the Family", en E. Berquó y P. Xenos (eds.), Family Systems and Cultural Change, Oxford University Press, p. 161-175.

(1965), "The Cohort as a Concept in the Study of Social Change", American Sociological Review, vol. 30, núm. 6, pp. 843-861.

Salles, V. (2001), "El debate micro-macro: dilemas y contextos", Perfiles Latinoamericanos, vol. 10, núm. 18, pp. 115-151.

Saltalamacchia, H. (1992), La historia de vida: reflexiones a partir de una experiencia de investigación, Caguas, Puerto Rico, CIJUP.

Settersten, R. y K. U. Mayer (1997), "The Measurement of Age, Age Structuring and the Life Course", Annual Review of Sociology, núm. 23, pp. 233261.

Slim, H. y P. Thompson (1993), Listening for a Change. Oral Testimony and Development, Londres, Panos.

Tashakkori, A. y C. Teddlie (1998), Mixed Methodology. Combining Qualitative and Quantitative Approaches, Thousand Oaks-Londres-Nueva Delhi, Sage (Applied Social Research Series, 46).

Zavala, M. E. (1992), Cambios de fecundidad en México y políticas de población, México, El Colegio de México/Fondo de Cultura Económica. 